\title{
Determination of Residues of two Aminoglycoside antibiotics and five Quinololine antibiotics and Their Metabolites in Penaeus vannamei by Ultra High Performance Liquid Chromatography Tandem Mass Spectrometry
}

\author{
ZHA Yu-bing ${ }^{1}$, YANG Chun-liang ${ }^{1,3}$, Lv Yi-zhi ${ }^{2}$, Ye Jian-zhi ${ }^{* 1,3}$, ZENG Shao-dong1,3, WANG Xiao-fang ${ }^{1 *}$, DENG \\ Xiao-hong ${ }^{2}$, MA Hui-fang ${ }^{1,3}$, PAN Xiaowei, ${ }^{1,3}$ LIN Li-yun ${ }^{1,3}$ \\ ${ }^{1}$ Agriculture Products Processing Research Institute of South China, Zhanjiang 524001, China \\ ${ }^{2}$ Agricultural technology extension Center, Zhanjiang 524001, China \\ ${ }^{3}$ Laboratory of Quality \& Safrty Risk Assessment on Agro-products Processing (Zhanjiang), Ministry of agriculture
}

\begin{abstract}
An ultra performance liquid chromatography - electrospray ionization triple-quadruple tandem mass spectrometric(UPLC-ESI MS /MS) method was developed for determining Quinololine antibiotics (Carbadox,,Quinocetone, Mequindox, Olaquindox, Cyadox, Tilmicosin, Tylosin, quinoxaline-2-carboxylic acid,3-methylquinoxaline-2-carboxylic acid) and aminoglycoside antibiotics(Tilmicosin,Tylosin) residues in Penaeus. vannamei, Samples were extracted with acidified acetonitrile, then cleaned up with hexane. The separation of targets was carried out on a Waters BEH C18 column using acetonitrile and $0.1 \%$ fomicacid as mobile phase. Analyte identification and quantification were performed by MS/MS under multiple-reaction monitering(MRM) mode with the external standard method. The results showed that there were good linear relationships between peak area and concentrations of nine targets in the range of $2.5 \sim$ $50 \mu \mathrm{g} / \mathrm{L}$, with correlation coefficients more than 0.999 and The limits of detection(LOD) were in the range of $0.1 \sim 0.9 \mu \mathrm{g} / \mathrm{kg}$. the recoveries ranged from $80.2 \% \sim 94.0 \%$, with RSDs of $1.7 \% \sim 8.4 \%$. The method is simple, accurate and precise, and It could satisfy the requirement of mass and fast analysis in the laborator.
\end{abstract}

\section{Introduction}

Quinoxalines are synthetic antimicrobial pro-growth agents. With the mother nuclear structure of Quinoxaline-1,4-Dioxide (Quinoxaline ring), the early representative products were carbadox and olaquindox. These drugs have good antibacterial and growth-promoting effects and are often added to feed and are widely used in livestock and poultry, aquaculture ${ }^{[1]}$. However, toxicology studies in recent years have proved that carbadox and olaquindox have different degrees of consistency, after the use of carbadox, livestock muscles and tissues will have different degrees of residues of carbadox and olaquindox as the representative of the residual problem of the drug has attracted the attention of international organizations and many countries and regions around the world. In 1998, the European Union banned carbadox, olaquindox and tylosin as feed additives added to the feed ${ }^{[2]}$. The U.S. only allows carbadox as a therapeutic drug. China's Ministry of Agriculture also made it clear at the end of 2001 and 2002 that: It is prohibited to use olaquindox as a growth promoter in poultry and aquaculture ${ }^{[3]}$. In 2006, the European Union also had new regulations prohibiting the addition of Quinoxaline to feed, and requiring that animal-sourced foods not be detected ${ }^{[4]}$.

The Ministry of Agriculture of the People's Republic of China No. 560 mentions that carbadox is also prohibited because safety issues affect animal food safety, public health and animal food exports in China. In view of the dual properties of the excellent antibacterial growth-promoting effects of quinoxaline drugs and their potential toxic and side effects, the researchers have developed new varieties that preserve its antibacterial pro-growth effect and effectively reduce the toxic side effect, including Methaquine, Quinocetone and Cyadox. Methaquine, Quinocetone and Cyadox, as feed drug additives for the upgrading of quinolium, have been widely used in animal husbandry and aquaculture production after nearly 20 years of series of safety evaluation, breeding tests and effect verification. It is expected to be a substitute for more toxic drugs such as quinoxaline, widely used in livestock and aquaculture.

Tilmicosin and Tylosin are macrolide antibiotics, can be used for the treatment of Erlan-positive bacteria and some Erlan-negative bacteria, mold, helix and mycogen, etc. have a very good antibacterial effect ${ }^{[5]}$. With the long-term widespread use of macrolide

\footnotetext{
*Corresponding author's e-mail: 76462276@qq.com
} 
antibiotics,Humans are increasingly recognizing the dangers of these drugs to humans ${ }^{[6]}$, Residues in animal food pose a great threat to food safety. Many countries in Europe and the United States have banned the addition of antibiotics to the production of food animals, and China has also established a maximum residue limit for these two drugs ${ }^{[7]}$.

At present, there are more single detection methods for Carbaxy, quinocetone, Acetoquine, quinolone, quinolone, cyadox, Tilmicosin, tylosin and its metabolites quinoxaline-2-carboxylic acid, 3-methylquinoxaline-2-carboxylic acid in Penaeus Vannamei.at most, four quinoxaline drugs can be determined simultaneously, mainly by chromatography ${ }^{[8-11]}$ and liquid chromatography tandem mass spectrometry ${ }^{[12-18]}$. However, the simultaneous determination of quinoxaline, quinoxaline metabolites and Macrolide residues in Penaeus Vannamei has not been reported. A method for the simultaneous determination of two Macrolide and five quinoxaline and their metabolites in Penaeus Vannamei by UPLC-MS/MS has been developed, the method can meet the requirements of simultaneous detection of the above 9 drugs.

\section{Materials and methods}

\subsection{Reagents and instruments.}

Carbadox, Quinocetone, Mequindox, Olaquindox, Cyadox, Tilmicosin, Tylosin, quinoxaline-2-carboxylic acid, 3-Methylquinoxaline-2-carboxylic acid.

Acetonitrile (chromatographic purity) ; methanol (Analytical Purity) ; formic acid (chromatographic purity) ; acetic acid (chromatographic purity) ; ammonium Acetate (chromatographic purity) ; Ethyl Acetate (Analytical Purity) ; water is secondary distilled water.

Waters ACQUITY UPLCTM - TQD MS/MS System (Waters, USA; Nitrogen Blower (Organomation, USA). ) ; Ultrasonic Cleaner (ELMA, Germany); Vortex Mixer (IKA, Germany); High-speed centrifders (Japan's Isachi); Milli - Q Pure Water Systems (Millipore, USA); Electronic Balance (Isazu Corporation).

\subsection{Standard solution preparation}

\subsubsection{Reserve liquid}

The reference standards of Carbaxy, quinocetone, Acetoquine, olaquindox, cyadox, Tilmicosin, tylosin, quinoxaline-2-carboxylic acid and 3-methylquinoxaline-2-carboxylic acid were each 10.0 $\mathrm{mg}$, respectively, acetonitrile was dissolved in $100 \mathrm{~mL}$ volumetric flask, and $100 \mu \mathrm{g} / \mathrm{mL}$ standard stock solution was prepared. The solution was stored at $-18{ }^{\circ} \mathrm{C}$ in the dark. The shelf life was 6 months. 2.2.2 Mixed the standard intermediate solution
The $1.0 \mu \mathrm{g} / \mathrm{mL}$ mixed standard intermediate solution was prepared from $0.1 \mathrm{~mL}$ reserve solution in $10 \mathrm{~mL}$ volumetric flask with acetonitrile to $10 \mathrm{~mL}$ and stored at $4{ }^{\circ} \mathrm{C}$.

\subsubsection{Mix standard work curve working fluid}

Accurately absorb a certain amount of the above-mentioned mixed standard intermediate solution, diluted with blank sample extract into the corresponding standard working fluid, ready for use.

\subsection{Pre-sample treatment}

$2.0 \mathrm{~g}$ sample was added into $50 \mathrm{~mL}$ centrifuge tube, $2 \mathrm{~g}$ anhydrous sodium sulfate and $10 \mathrm{~mL} 0.1 \%$ formic acid-acetonitrile solution were added, then the sample was vortex mixed for $1 \mathrm{Min}$, and the sample was extracted by ultrasonic wave for $5 \mathrm{~min}$. The supernatant was centrifuged for $5 \mathrm{~min}$ at $8000 \mathrm{R} / \mathrm{min}$, and the residue was extracted once with $10 \mathrm{~mL} \quad 0.1 \%$ formic acid-acetonitrile solution, then mixed with the two extractions, and the supernatant was blown to near-dry by nitrogen at $35^{\circ} \mathrm{C}$.

Add $1.0 \mathrm{~mL}$ of the residue dissolved in the initial mobile phase, add $2.0 \mathrm{~mL}$ of acetonitrile saturated n-hexane, swirl mix it for $30 \mathrm{~s}$, transfer it to $5 \mathrm{~mL}$ plug centrifuge tube, centrifuge for $12000 \mathrm{R} / \mathrm{min}$ for $5 \mathrm{~min}$, discard the supernatant, remove the supernatant, remove the supernatant and filter it through $0.2 \mu \mathrm{m}$ membrane for LC-MS/MS determination.

\subsection{Chromatography conditions}

Column: UPLC ACQUITY BEH, 50 x $2.1 \mathrm{~mm}, 1.7 \mu \mathrm{m}$; The flow phase: Acetylene (A)/0.1 methamphetate solution $(\mathrm{V}: \mathrm{V})$; The gradient ebasion procedure is : 0 to $1.0 \mathrm{~min}, 90 \% \mathrm{~A} ; 1$ to $2.0 \mathrm{~min}, 90 \%-50 \% \mathrm{~A} ; 2.0 \sim$ to $3.6 \mathrm{~min}$, $50 \%-10 \% \mathrm{~A}$; 3.6 to $4.5 \mathrm{~min}, 10 \%-90 \% \mathrm{~A} ; 4.5$ to $5.5 \mathrm{~min}$, $90 \%$ A hold $1.0 \mathrm{~min}$. Flow rate: $0.3 \mathrm{~mL} / \mathrm{min}$; Column temperature: $30{ }^{\circ} \mathrm{C}$; The amount of samples: $10 \mu \mathrm{L}$.

\subsection{Mass spectrometry conditions}

Ion source: ESI, positive ion mode, capillary voltage: 4.0 $\mathrm{kv}$, ionsource temperature: $110{ }^{\circ} \mathrm{C}$, desolate gas temperature $350{ }^{\circ} \mathrm{C}$;Desolvent gas flow: $800 \mathrm{~L} / \mathrm{hr}$; tapered air flow $50 \mathrm{~L} / \mathrm{hr}$; Taper voltage: $40 \mathrm{v}$; Scan mode: Multi-reaction monitoring (MRM). For qualitative and quantitative ion pairs and related mass spectrometry parameters, see Table 1.

\section{Results and discussions}

\subsection{The choice of extracting solvents}

Both quinoxaline drugs and macrolide antibiotics are susceptible to polar solvents. Such as methanol, acetonitrile, formic acid acetonitrile, methioninewater 
and acetonitrile water as extraction solvents. In the course of the experiment, the choice of solvent directly affects the accuracy of the method. In this experiment, methanol, acetonitrile, formic acid/acetonitrile, methanol/water and acetonitrile/ water were selected as extraction solvent. The results showed that $0.1 \%$ formic acid/acetonitrile system had better comprehensive effect on the extraction of two kinds of targets, and the recovery rate and spectrum were better than other extraction solvents. Adding proper amount of anhydrous sodium sulfate before adding the extract solution can adsorb the water in the sample, which is helpful to improve the extraction efficiency of the sample.

\subsection{Selection of liquid chromatography conditions}

In the analysis of quinoxaline and Macrolide residues in poultry and Penaeus Vannamei, the $\mathrm{C} 18$ column was selected, and the UPLC ACQUITY Beh C18 column was used. Because the composition and proportion of the mobile phase play a key role in the separation of the target, the composition and proportion of the mobile phase are mainly studied. When acetonitrile was used as organic phase, $0.1 \%$ formic acid, $0.1 \%$ acetic acid and 5 $\mathrm{mM}$ ammonium acetate solution were added to the aqueous phase, the results showed that the quinocetone and cyadox had poor shape and low sensitivity, when formic acid is added into the water phase, the target is easy to be protonated and has a positive charge, thus the ionization efficiency is improved and the sensitivity of the method is improved. Therefore, $0.1 \%$ formic acid solution-acetonitrile as the mobile phase, gradient elution, 9 kinds of target can be obtained better separation effect, peak symmetry, retention time is moderate.

\subsection{Selection of mass spectrometry conditions}

Tab 1 The optimization parameters of MRM of the analytes

\begin{tabular}{|c|c|c|c|c|c|}
\hline compound & $\begin{array}{l}\text { Retention } \\
\text { time }(\mathrm{t} / \mathrm{min})\end{array}$ & Parent ion $(\mathrm{m} / \mathrm{z})$ & $\begin{array}{l}\text { Daughter } \\
\text { ions }(\mathrm{m} / \mathrm{z})\end{array}$ & $\begin{array}{c}\text { Cone } \\
\text { voltage }(\mathrm{V})\end{array}$ & $\begin{array}{c}\text { Collision } \\
\text { energy }(\mathrm{eV})\end{array}$ \\
\hline carbadox & 1.38 & 263.1 & $229.1 / 231.1^{*}$ & 30 & $15 / 15$ \\
\hline methaquine & 1.36 & 219.1 & $160.1 / 185.1^{*}$ & 30 & $15 / 15$ \\
\hline quinocetone & 1.71 & 307 & $197 / 273^{*}$ & 30 & $25 / 25$ \\
\hline olaquindox & 1.31 & 264 & $143 / 212^{*}$ & 30 & $40 / 25$ \\
\hline cyadox & 1.80 & 272.1 & $143.1 / 188.1^{*}$ & 25 & $15 / 15$ \\
\hline $\begin{array}{l}\text { quinoxaline-2-car } \\
\text { boxylic acid }\end{array}$ & 1.59 & 175.2 & $129.2 / 131.2^{*}$ & 25 & $12 / 12$ \\
\hline MQCA & 1.65 & 189 & $143.1 / 145.1^{*}$ & 18 & $15 / 15$ \\
\hline tlmicosin & 1.13 & 869.5 & $132.2 / 174.2$ & 30 & $55 / 50$ \\
\hline tylosin & 1.84 & 916.5 & $101.1 / 174.1$ & 57 & $45 / 40$ \\
\hline
\end{tabular}

\subsection{The linear relationship and check-out limit of the method}

Considering the Matrix effect, the standard solution was added to the blankMatrix as the standard curve, and a series of mixed standard working solutions wereprepared with the concentration of $2.5,5.0,10.0,20.0,50.0 \mu \mathrm{g} / \mathrm{L}$ respectively. The sample was injected from low to high in the order of the concentration, and the analysis was carried out under the above conditions. The concentration (x) was linear regressed by the peak area (Y) of the tested components. The results showed a good linear relationship (see table 2). The detection limits of

According to the structure of quinoxaline and Macrolide drugs, Esi $(+)$ was selected as the ionization mode, and a
single standard sample was injected by flow injection compounds with concentration of $100 \mathrm{ng} / \mathrm{mL}$ were cyadox, Tilmicosin, tylosin, quinoxaline-2-carboxylic acid, 3-methylquinoxaline-2-carboxylic acid were determined to be 263.1, 307.0, 219.1, 264.0, 272.1, 869.5 were selected by means of Ion scanning, and the collision energy was increased gradually. With the decrease of parent ion abundance, the ion abundance increased
gradually. Two ions with the greatest abundance of each selected as quantitative ions. At the same time, the temperature, Collision are optimized. Optimize the impact voltage and other mass spectrometry analysis conditions (see table 1) . 


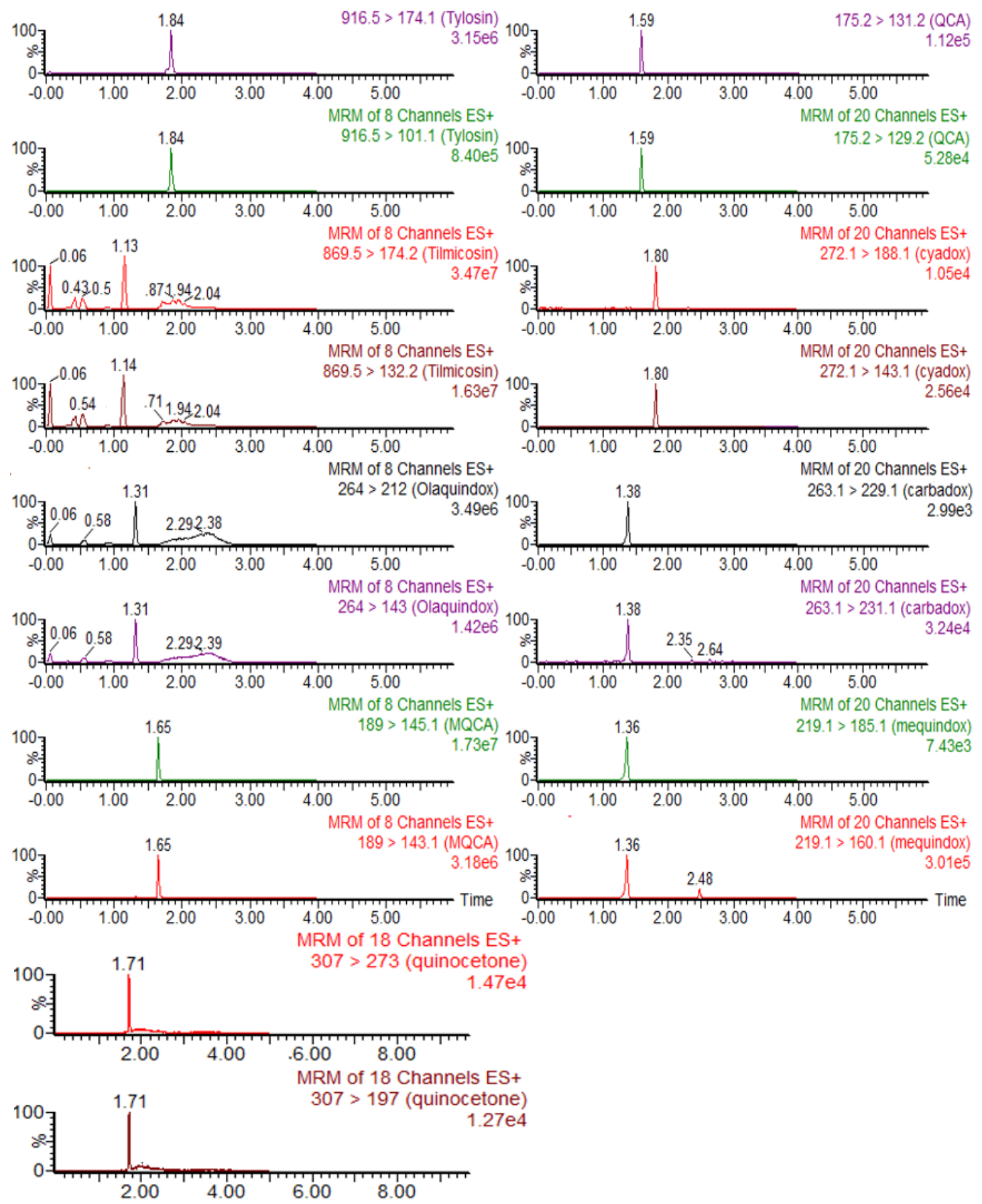

Figure. 1. MRM diagram of 9 compounds mixing standard solutions. MRM.

Table 2. Linear range, regression equatio, correlation coeficientfor target compounds

\begin{tabular}{ccccc}
\hline compound & Regression equation & $\begin{array}{c}\text { Linear } \\
\text { range }(\mu \mathrm{g} / \mathrm{L})\end{array}$ & $\begin{array}{c}\text { Correlation } \\
\text { coefficient }\end{array}$ & $\begin{array}{c}\text { The detection } \\
\text { limit }(\mu \mathrm{g} / \mathrm{kg})\end{array}$ \\
\hline carbadox & $\mathrm{y}=460.1 \mathrm{x}+230$ & $2.5-50$ & 0.9993 & 0.3 \\
methaquine & $\mathrm{y}=725.5 \mathrm{x}-185.9$ & $2.5-50$ & 0.9992 & 0.4 \\
quinocetone & $\mathrm{y}=480.2 \mathrm{x}+220.0$ & $2.5-50$ & 0.9996 & 0.4 \\
olaquindox & $\mathrm{y}=203.6 \mathrm{x}+93.2$ & $2.5-50$ & 0.9997 & 0.1 \\
$\begin{array}{c}\text { cyadox } \\
\text { quinoxaline-2-c }\end{array}$ & $\mathrm{y}=1010.3 \mathrm{x}+345.5$ & $2.5-50$ & 0.9995 & 0.9 \\
arboxylic acid & $\mathrm{y}=738.2 \mathrm{x}+129.5$ & $2.5-50$ & 0.9992 & 0.3 \\
MQCA & $\mathrm{y}=306.4 \mathrm{x}+22.8$ & $2.5-50$ & 0.9991 & 0.1 \\
\hline
\end{tabular}




\begin{tabular}{cllll}
\hline tlmicosin & $\mathrm{y}=1250.1 \mathrm{x}+301$ & $2.5-50$ & 0.9995 & 0.1 \\
tylosin & $\mathrm{y}=460.2 \mathrm{x}+49.5$ & $2.5-50$ & 0.9993 & 0.1 \\
\hline
\end{tabular}

Table 3 The recovery rate and precision determination of 9 compounds in the sample(n-6).

\begin{tabular}{ccccc}
\hline (Compund) & $\begin{array}{c}\text { Spiked } \\
(\mu \mathrm{g} / \mathrm{kg})\end{array}$ & $\begin{array}{c}\text { Measured } \\
(\mu \mathrm{g} / \mathrm{kg})\end{array}$ & Recovery $(\%)$ & RSD $(\%)$ \\
\hline \multirow{3}{*}{ carbadox } & 2 & 1.85 & 92.5 & 3.7 \\
& 10 & 9.27 & 92.7 & 4.5 \\
methaquine & 50 & 44.2 & 88.4 & 2.8 \\
& 2 & 1.88 & 94.0 & 6.2 \\
quinocetone & 10 & 8.52 & 85.2 & 3.9 \\
& 50 & 43.6 & 87.2 & 2.1 \\
olaquindox & 2 & 1.78 & 89.0 & 3.5 \\
& 10 & 8.63 & 86.3 & 6.4 \\
cyadox & 50 & 44.0 & 88.0 & 5.2 \\
& 2 & 1.81 & 90.5 & 3.3 \\
quinoxaline-2-ca & 10 & 8.20 & 82.0 & 5.2 \\
rboxylic acid & 50 & 40.1 & 80.2 & 1.7 \\
& 2 & 1.75 & 87.5 & 4.6 \\
& 10 & 8.13 & 81.3 & 2.1 \\
MQCA & 50 & 40.3 & 80.6 & 5.5 \\
& 2 & 1.70 & 85.0 & 6.9 \\
tlmicosin & 50 & 8.32 & 83.2 & 4.2 \\
& & 42.6 & 85.2 & 4.6 \\
& 2 & & & 1.9 \\
& 10 & 1.69 & 84.5 & 4.2 \\
& 50 & 8.78 & 87.8 & 2.8 \\
& 2 & 40.8 & 81.6 & 7.2 \\
& 10 & 1.85 & 92.5 & 3.9 \\
& 50 & 9.00 & 90.0 & 2.7 \\
\hline
\end{tabular}

Carbaxy, quinocetone, acetyl-methyl-quinolone, quinolone, cyadox, Tilmicosin, tylosin, quinoxaline-2-carboxylic acid and 3-methyl-quinoxaline-2-carboxylic acid are shown in table 2 .

\subsection{Method accuracy and precision.}

Taking Penaeus Vannamei as the object of study, the experiment of recovery by adding standard was carried out, and three different levels of addition $(2 \mathrm{ug} / \mathrm{kg}, 5 \mathrm{ug} / \mathrm{kg}$ and $20 \mathrm{ug} / \mathrm{kg}$ ) were set, and six parallel experiments were set for each concentration, in order to study the accuracy and precision of the determination results under different standard addition levels. The recoveries and their relative standard deviations were calculated, and the results are shown in Table 3. The results showed that the recovery of the additive was $80.2 \% \sim 94.0 \%$, the RSD was $1.7 \% \sim 8.4 \%$, and the detection limit was $0.1 \sim 0.9$ $\mathrm{g} / \mathrm{kg}$. The results show that the analytical method has good accuracy and precision. repeatability, accuracy, precision and sensitivity, and meets the performance requirements of the residue analysis method, the method is suitable for routine analysis of residues of quinoxaline and Macrolide and their main metabolites in Litopenaeus Vannamei and can be used for simultaneous determination of two kinds of Macrolide, five kinds of quinoxaline and two kinds of their main metabolites, a more accurate and comprehensive analysis of quinoxaline and Macrolide residues can be performed than previously reported methods for the determination of quinoxaline and Macrolide.

\section{Conclusions}

HPLC-MS/MS method was established for the determination of Quinoxaline, quinoxaline metabolites and Macrolide in Penaeus Vannamei. The pretreatment method and the conditions of chromatography and mass spectrometry were studied. The average recovery was $80.2 \%-94.0 \%$, RSD was $1.7 \%-8.4 \%$, and the 
detection limit was $0.1-0.9 \mathrm{~g} / \mathrm{kg}$. The method has the characteristics of good

\section{Acknowledgments}

This work was supported by the National Program for Quality and Safety Risk Assessment of Agricultural Products of China (GJFP2019012, GJFP2019013, GJFP2019019, GJFP2019038); Central Public-interest Scientific Institution Basal Research Fund for Chinese Academy of Tropical Agricultural Sciences ( No. 1630122017020); the Natural Science Foundation of Hainan Province (No.219QN290).

\section{References}

1. Cao,Y.Z., Tension, Liang,J.P., et al. Niobium-1,4-oxides.Advances in the study of special toxicology of antimicrobial pro-growth agents[J]. Advances in animal medicine. 2001, 229(2):17-20.

2. Situ,C., Elliott, C.T., Simultaneous and rapid detection of five banned antibiotic growth promoters by immunoassay[J]. Anal Chim Act, 2005,529(1/2): 89-96.

3. He,Q.H., Study of skin photosensitive toxicity and long-term toxicity of quinxado[D]. Huahua Agricultural University, 2005.

4. Wu, C. M., Li,Y., Shen,J. Z., et al. LC-MS-MS Quantification of four quinoxaline-1, 4-dioxides in swine feed[J]. Chromatographia,2009,70(11/12):1605-1611.

5. Wang, X.Z., Liu,G.Y., North lycomycin prevention and treatment of grass fish tri-disease test (J) freshwater fisheries, 1994, 24(6): 18-20.

6. Yang, F., Li, Y.p., Fang,Y., et al. Physical and Chemical Inspection - Chemistry Book, 2007, 43 (4): 272.

7. Ministry of Agriculture of the People's Republic of China Announced No. 235 Maximum Residual Limits of Veterinary Medicine in Animal Foods[S]. 2002.

8. Croteau,D.,Vallee,F.,Bergeron,M.G., et al. High-performance liquid chromatographic assay of erythromycin and esters using electrochemical detection[J]. J Chromatogr B, 1987, 419: 205-212.

9. Berrada,H., Borrull,F., Font,G., et al. Validation of a confirmatory method for the determination of macrolides in liver and kidney animal tissues in accordance with the European Union regulation
2002/657/EC[J]. Journal of Chromatography A, 2007,1157(1/2): 281-288.

10. Yin,G., Yu,M., Shou,C.J., et al. Residual detection of olaquindox, cabardo and its metabolites in chicken.[J]. Chinese Journal of Veterinary Medicine, 2006, 40(1):11-15.

11. Jia,Z.M., Wu,N.P., Song,Z.C., et al. High-efficiency liquid chromatography method to determine the chinese veterinary drug preparations in the drug[J].Chinese Journal of Veterinary Medicine[J].2010,44(3):13-15.

12. Liu,Y.c., Xu,W.h., Yu,L.l., et al. Solid phase extraction fluid chromatography - Mass spectrometry/mass spectrometry with the determination of large ring ester antibiotics in river water[J] .Journal of Analytical Testing.2006, 25(2):1-5.

13. Xia,M., Jia,L., Ji,Y.p., Liquid chromatography - Mass spectrometry simultaneously detects five large ring-endosteride antibiotics in livestock and poultry meat.[J] .Journal of Analytical Testing.2004, 23(Increase.):217 -222.

14. Liu,Y.T., Liu.Z.H., Ding,Y.M., HPLC-MS/MS for the simultaneous determination of quinocetone, olaquinol and five macrolide residues in penaeus vannamei, 29(8) : 44-47.

15. Pang,G.F., Xie,L.Q., Ou,Y.S., et al. Pang Guofang, Xie Liqi, Ou Yangshan, etc. . GB/T 20746-2006 determination of Carbaxy, olaquindox and metabolites in beef, pig liver and muscle by liquid chromatography-tandem mass spectrometry[s]. BEIJING: China Standard Press, 2006:1-6.

16. Dong,Y.C., Zhang,L.F., Zhang,K.Y.,et al. High-efficiency liquid chromatography - series mass spectrometry to detect residues of sorbets in pig and chicken eatable tissues.[J]. Chinese Journal of Veterinary Medicine . 2010,30(1):110-114.

17. Xu,Y.J., Ren,C.B., Tian,X.H.,et al. Ultra-efficient liquid chromatography, series mass spectrometry to determine carbadox, quinone, acetylmethasone and its metabolites in White Shrimps in South America[J]..Journal of Analytical Testing.2011,30(10):1133-1137.

18. Liu,F.Q., Zeng,M.H., Xu,S.F. et al. At the same time, modern agricultural science and technology are used to determine the drug methods in acetylene, oleolaquindox and quinone 3 in feed[J].2008,24:231-233. 